\title{
Hernia diafragmática de Bochdalek en un adulto
}

\author{
Bochdalek's diaphragmatic hernia in an adult \\ Carlos Tadeo Perzabal Aviléz, * César Alberto López Jaime, ${ }^{\ddagger}$ \\ Emmanuel Colín Núñez, ${ }^{\S}$ Diana Laura Carrera Cruz ${ }^{\S}$ \\ Citar como: Perzabal ACT, López JCA, Colín NE, Carrera CDL. Hernia diafragmática de Bochdalek en \\ un adulto. Acta Med GA. 2022; 20 (1): 105-107. https://dx.doi.org/10.35366/103570
}

Mujer de 17 años que acude tras presentar cuadro de dolor de dos años de evolución en flanco izquierdo que se irradia a hipocondrio izquierdo de tipo opresivo, que se acompaña de disnea. A la exploración física con hipoventilación basal izquierda, abdomen blando, depresible, doloroso a la palpación profunda en hipocondrio izquierdo, sin datos de irritación peritoneal.

Radiografía de tórax a su ingreso con imagen radiopaca basal izquierda con niveles hidroaéreos (Figura 1), por lo que se solicita estudio tomográfico que confirma la pre- sencia de una hernia diafragmática izquierda con colon, bazo, estómago e intestino delgado dentro de su contenido (Figura 2).

Se pasa a sala de operaciones en donde se realiza incisión paramedia izquierda, encontrando orificio herniario de $5 \times 5 \mathrm{~cm}$, en su interior se observa colon, bazo, estómago e intestino delgado, se reduce dicho contenido y se reseca el saco herniario, se realiza cierre primario del defecto diafragmático y previo a la colocación de la última sutura se colocó sonda endopleural y se solicitó

Figura 1:

Radiografía de tórax y abdomen con imagen radiopaca en región basal pulmonar izquierda que corresponde a niveles hidroaéreos.

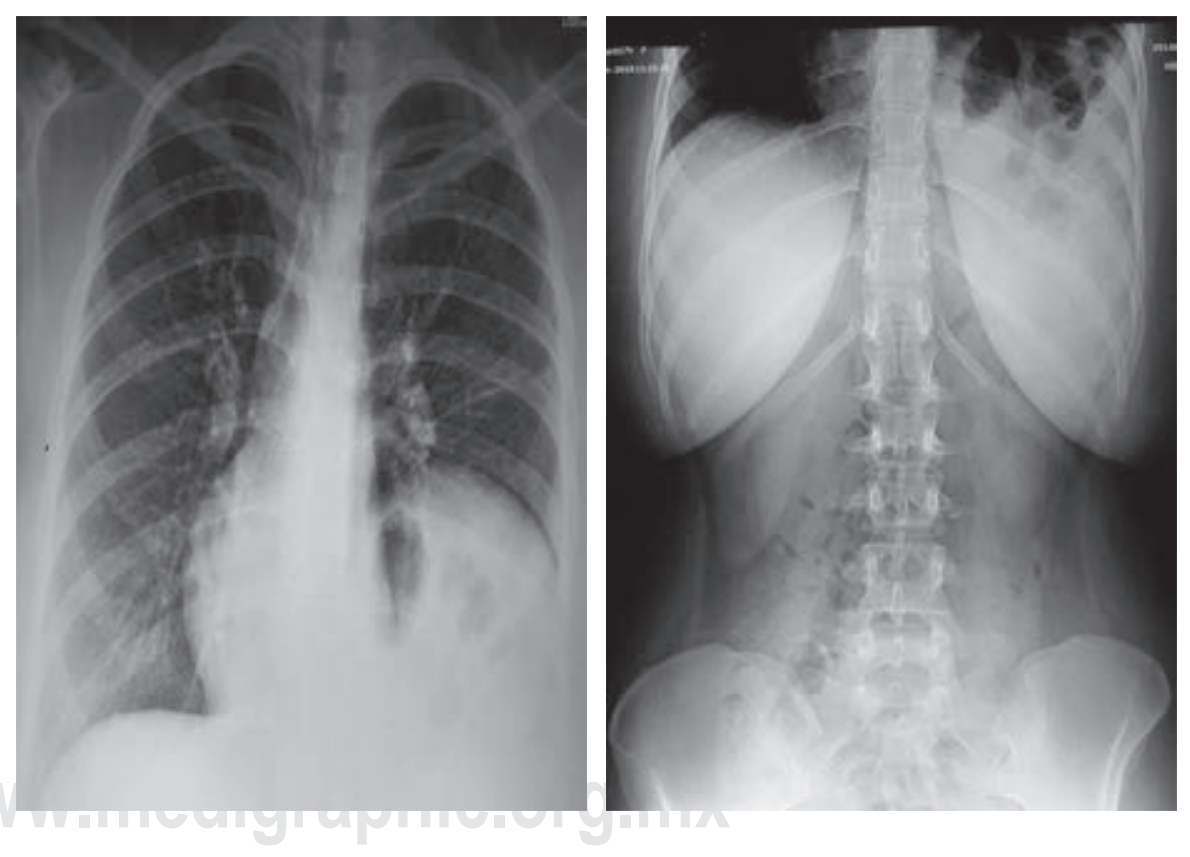

* Cirujano General y Laparoscopia.

‡ Médico Residente de Cirugía General.

$\S$ Médico General.

Hospital General de Ciudad Juárez. Cd. Juárez, Chihuahua.
Correspondencia:

César Alberto López Jaime

Correo electrónico: cesarlopezj91@gmail.com

Aceptado: 03-08-2020.

www.medigraphic.com/actamedica 
Figura 2:

Tomografía computarizada toracoabdominal en corte coronal con evidencia de hernia diafragmática izquierda, con estómago, colon, intestino delgado y bazo en su interior.
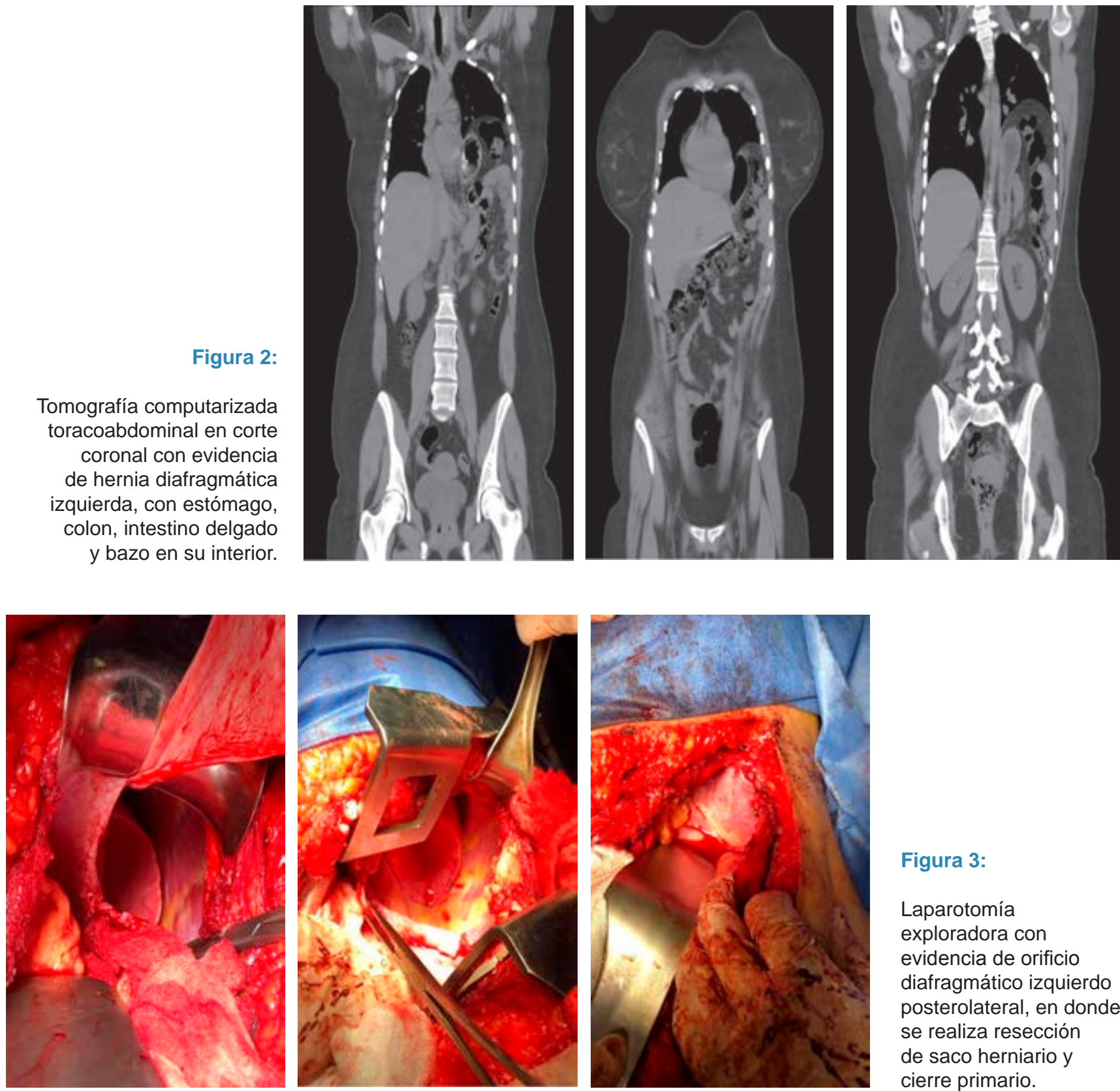

Figura 3:

\section{Laparotomía \\ exploradora con \\ evidencia de orificio \\ diafragmático izquierdo \\ posterolateral, en donde \\ se realiza resección \\ de saco herniario y \\ cierre primario.}

la realización de múltiples maniobras de Valsalva por el servicio de anestesiología, para después cerrar de manera simultánea el defecto mientras se retiraba la sonda endopleural, se cierra por planos y se da por terminado el procedimiento (Figura 3).

Se solicita radiografía de tórax de control, con evidencia de atelectasia apical derecha, con neumotórax basal mínimo izquierdo, por lo cual se egresa a la paciente para manejo por consulta externa a las 48 horas de su postoperatorio (Figura 4).

\section{HERNIA DE BOCHDALEK}

Descrita por Vincent Alexander Bochdalek en 1848,,$^{1-3}$ aunque en 1754 McCauley describe el curso clínico y la anatomía post mortem de un infante. ${ }^{2}$ Kirkland en 1959 publicó por primera vez una revisión de 34 casos de hernias de Bochdalek en adultos. ${ }^{1}$

En la actualidad, no se ha explicado de manera clara la patogenia de las hernias diafragmáticas. Se asocia de 


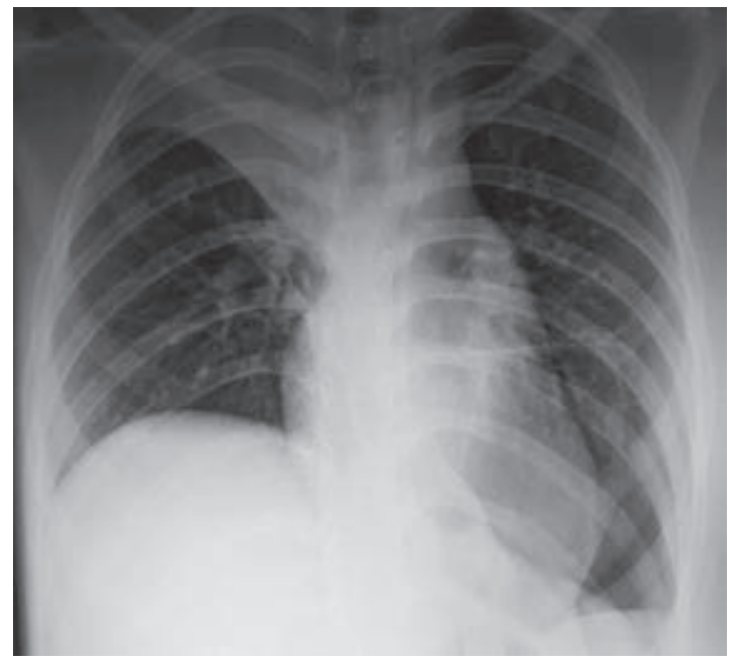

Figura 4: Radiografía de tórax en el postoperatorio inmediato sin evidencia de neumotórax residual y sin imagen radiopaca basal izquierda en comparación a estudio de ingreso.

un $10-30 \%$ con un defecto cromosomal, siendo la causa etiológica desconocida en un $70 \%$ de los casos. ${ }^{4}$

El diagnóstico es difícil, ya que la presencia de esta enfermedad es demasiado rara en la edad adulta, por los diagnósticos prenatales y la mortalidad elevada. Si no se llega a un diagnóstico adecuado, las complicaciones son vólvulos, incarceración, estrangulación, hemorragia o perforación de víscera, hasta la muerte. ${ }^{1,3}$

Las presentaciones clínicas de la hernia de Bochdalek en los adultos a comparación de los pacientes en edad pediátrica son diferentes; en adultos pueden ser dolor torácico, intolerancia a los alimentos con vómito postprandial, reflujo gastroesofágico, disnea, dolor abdominal y datos de oclusión intestinal. ${ }^{1,5}$

Se puede realizar el diagnóstico con los estudios convencionales, siendo la radiografía y los estudios con bario una opción en el adulto. ${ }^{3}$ El uso de la tomografía computarizada es necesaria para realizar un diagnóstico correcto, ya que un 38\% de los adultos con hernia de Bochdalek son diagnosticados con derrame pleural, empiema, neumotórax o quiste pulmonar. ${ }^{1,2}$

El manejo quirúrgico está indicado en todos los casos con diagnóstico de hernia diafragmática por las repercusiones potenciales que pueden ocurrir con el paso del tiempo. Existen diferentes tipos, los cuales se abordan con el tratamiento de laparotomía, laparoscopia, toracotomía, toracoscopia o una combinación de éstas. ${ }^{5}$

\section{REFERENCIAS}

1. Alam A, Chander BN. Adult Bochdalek hernia. Med J Armed Forces India. 2005; 61 (3): 284-286.

2. Brown SR, Horton JD, Trivette E, Hofmann LJ, Johnson JM. Bochdalek hernia in the adult: demographics, presentation, and surgical management. Hernia. 2011; 15 (1): 23-30.

3. Schumacher L, Gilbert S. Congenital diaphragmatic hernia in the adult. Thorac Surg Clin. 2009; 19 (4): 469-472.

4. Kosinski P, Wielgos M. Congenital diaphragmatic hernia: pathogenesis, prenatal diagnosis and management - literature review. Ginekol Pol. 2017; 88 (1): 24-30.

5. Ooi G, Lloyd D. Symptomatic congenital diaphragmatic hernia following right nephrectomy. ANZ J Surg. 2017; 87 (3): 198-199. 